\title{
Every Posterior Fossa Mass Is Not a Tumor- Rare Case Report of Isolated Intracranial Infantile Myofibromatosis
}

\author{
Kishor Taori, Mansi Jain*, Lalit Garg, Ajinky Patil, Jawhar Rathod, Rohit Khisti, \\ Amit Disawal, Ramesh Parate, Shyam Chhadi
}

Department of Radiodiagnosis, Government Medical College, Nagpur, India

Email: ${ }^{*}$ mansijainkmc4@gmail.com

Received 4 April 2014; revised 14 May 2014; accepted 2 July 2014

Copyright (C) 2014 by authors and OALib.

This work is licensed under the Creative Commons Attribution International License (CC BY).

http://creativecommons.org/licenses/by/4.0/

(c) (i) Open Access

\section{Abstract}

Infantile myofibromatosis is the most common fibrous disorder of infancy and early childhood. It can present in three forms-solitary lesion, multicentric with visceral involvement and multi-centeric without visceral involvement. Intracranial involvement is rare and when it occurs, it is generally extension of extracranial lesion into the intracranial compartment. Here we present a rare case of isolated posterior fossa involvement presenting clinically as congenital facial palsy.

\section{Keywords}

Infantile Myofibromatosis, Posterior Fossa, Congenital Facial Palsy

Subject Areas: Neurology, Radiology \& Medical Imaging

\section{Introduction}

Posterior fossa masses in infants are not a rare clinical entity. Clinical presentation can be variable depending on the location and extent of involvement of this critical space. We should be aware of the fact that not every mass in poaterior fossa is malignant. We report a rare case of infantile myofibromatosis in a neonate presenting with congenital facial palsy due to posterior fossa mass. This entity can present in various locations like skin, muscle, bone, subcutaneous and viscera. Intracranial involvement is rare with only few cases of isolated posterior fossa involvement reported in literature so far [1].

Final confirmation of this entity is by histopathological diagnosis with prognosis and treatment depending on extent and location of involvement.

${ }^{*}$ Corresponding author.

How to cite this paper: Taori, K., et al. (2014) Every Posterior Fossa Mass Is Not a Tumor-Rare Case Report of Isolated Intracranial Infantile Myofibromatosis. Open Access Library Journal, 1: e670. http://dx.doi.org/10.4236/oalib.1100670 


\section{Case Report}

A 10-day-old male neonate delivered at term was brought to our hospital with complaints of congenital right facial plasy. There was no contributory ante natal history or known inherited familial disease. On initial physical examination, neurological examination was normal. Neurosonogram was done as screening modality which revealed well defined hyperechoic lesion in posterior fossa on right side without any hydrocephalous or cystic encephlomalacic changes (Figure 1). CT head was performed to rule out the possibility of subdural hematoma. CT head plain study (Figure 2) revealed hyperdense extraaxial mass lesion in right cerebellopontine angle with punctate calcifications and erosion of pertrous temporal bone. There was no obstruction of the ventricular system. Superficial cerebral venous sinuses were dilated and hyperdense. MRI study was advised for further characterisation to rule out the possibility of neplastic lesion. On MRI there was a well defined extra-axial solid mass lesion in posterior fossa with epicenter in right cerebello-pontine and medullary-pontine angle of approximate size $3 \times 3 \times 2.4 \mathrm{~cm}$ in transverse AP and cranio-caudal axis. It was heterogeneously isointense on T1W, T2W \& FLAIR (Figure 3) with multiple interspersed tiny foci appearing hypointense on T2W and hyperintense on T1W. On Diffusion weighted imaging (Figure 4) the lesion was isointese to brain Parenchyma. On post contrast study (Figure 5) there was strong relatively homogenous enhancement. Medially the lesion was causing mass effect in

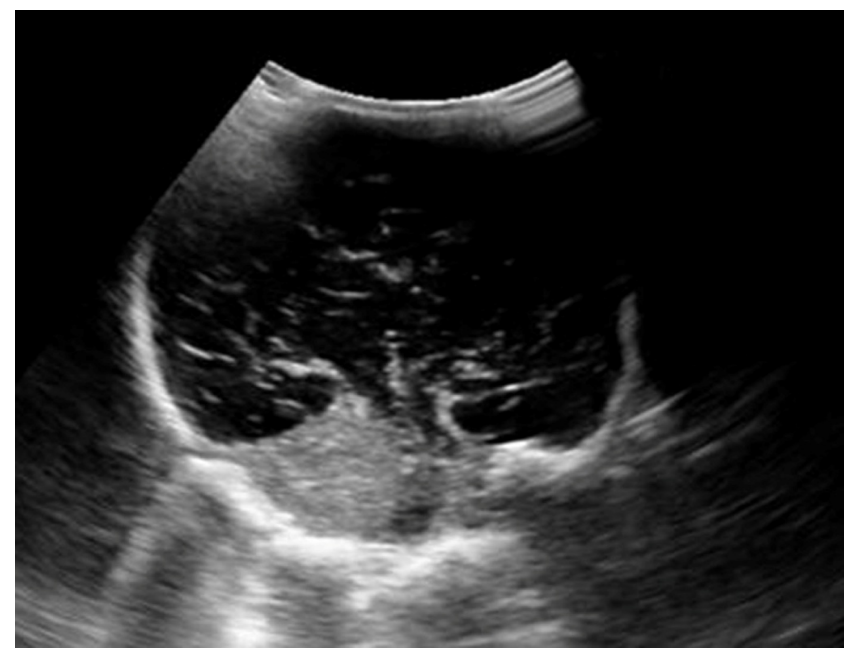

Figure 1. NSG reveals well defined hyperechoic lesion in posterior fossa on right side.

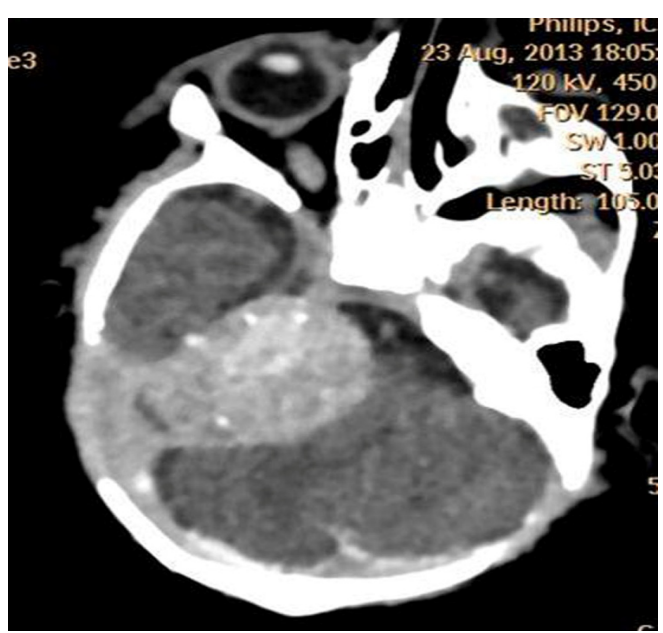

(a)

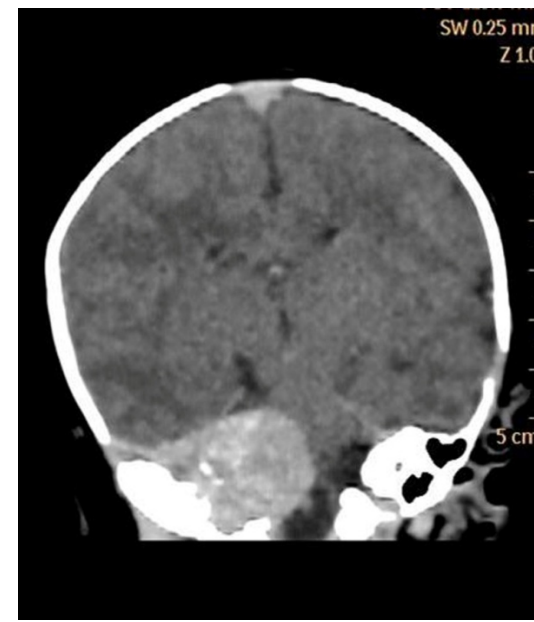

(b)

Figure 2. CT head palin reveals hyperdense extraaxial mass lesion in right cerebellopontine angle with punctate calcifications and erosion of pertrous temporal bone. 


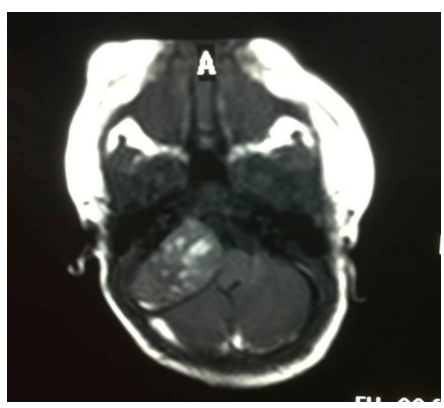

(a)

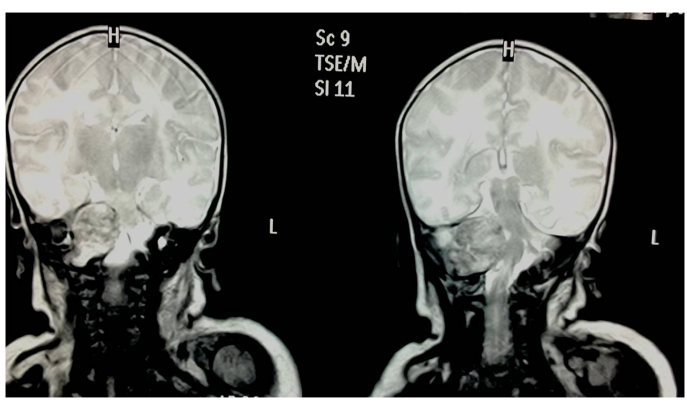

(b)

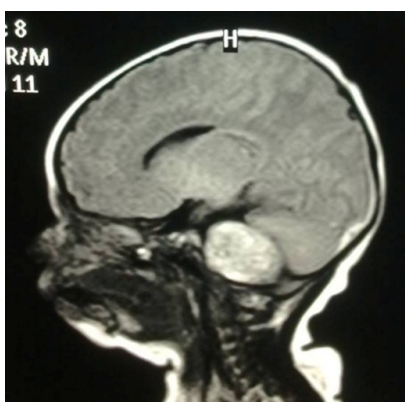

(c)

Figure 3. TIW, T2W and T2 FLAIR sequences reveal well defined extra-axial solid mass lesion in posterior fossa in right cerebello-pontine and medullary-pontine angle which appears heterogeneously isointense on T1W, T2W \& FLAIR and multiple interspersed tiny foci appearing hypointense on T2W and hyperintense on T1W. There is transjugular extension also noted on coronal sections.

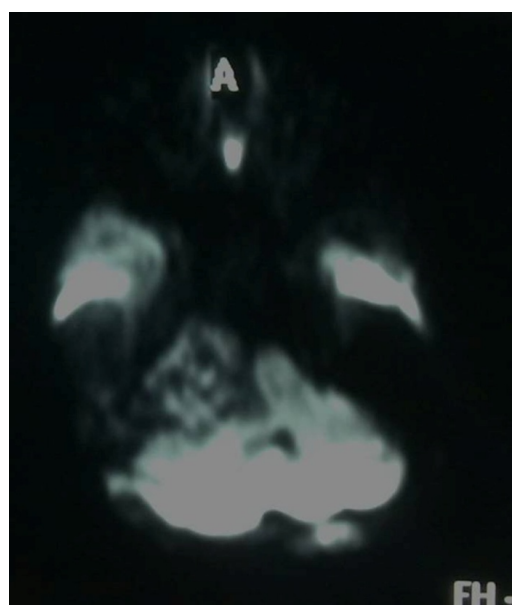

Figure 4. DWI sequence reveals hyperintensity s/o restrcited diffusion.

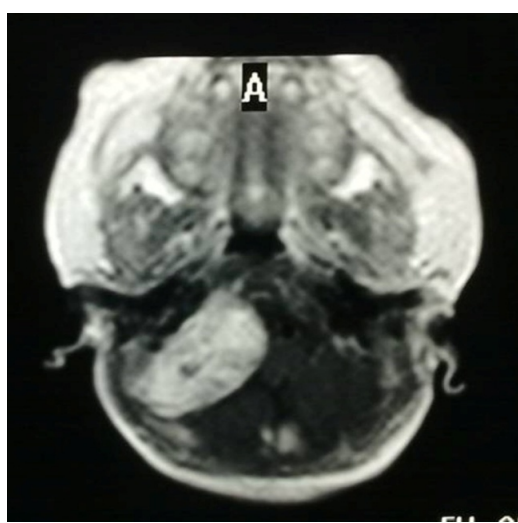

Figure 5. Post contrast T1W shows homogenously strong enhancement.

form of displacement and compression of the right cerebellar hemisphere, pons, medulla \& 4th ventricle without any e/o hydrocephalous in present scan. There was compression of the sigmoid sinus with venous sinuses and deep cortical veins appearing hyperintense on T1W possibly due to stasis with adequate contrast opacification on post-contrast T1 3D sequences. Inferiorly there was widening of the jugular foramen with transjuglar extension extra-cranially. 
We gave the possibility of infantile myofibroma of posterior fossa as most likely diagnosis over other more common lesions in this location like primitive neuroectodrmal tumor and a typical teratoid rhabdoid tumor. Imaging features favouring the possibility was isointensity of the lesion to brain parenchyma on diffusion weighted sequence. Skeletal survey and ultrasonography of abdomen was done which did not reveal any significant abnormality.

The mass in our case possibly arose from the lateral dural margin directly invaded the right sigmoid sinus and eroded the petrous temporal bone. The MR images confirmed the obliteration of the sigmoid sinus and enlargement of the jugular foramen. Supra-tentorial brain parenchyma showed normal gray-white matter.

Patient was referred to neurosurgery where biopsy was performed and specimen was pathlogically conrfimed to be consistent with imaging diagnosis of infantile myofibroma revealing whorls of spindle shaped cells of myofibroblastic origin. Subtotal resection was performed. Care was taken not to avoid damage to the lower cranial nerves, and resection was not extended into the carotid sheath or internal jugular vein.

Follow-up imaging (Figure 6) at 3 months after resection revealed significant reduction in size of the lesion, consistent with the natural history of this rare entity.

\section{Discussion}

Infantile myofibromatosis which is the most common fibrous disorder of infancy and early childhood was first described by Stout in 1954 [2]. It is more commonly seen in males. However, exact incidence is not known due to variable and unpredictable natural course of the disease. It is a disease with variable prognosis depending on type and extent of involvement. It can present in three forms-solitary lesion, multicentric lesions with visceral involvement and multicenteric lesions without visceral involvement. Multicenteric lesions can occur in skin, muscle, bone, subcutaneous tissue and viscera. More the visceral involvement worse is the outcome. The common sites with visceral involvement were lung, heart, gastrointestinal tarct, pancreas and liver. However, lesions have rarely been noted in intracranial compartment which are usually due to extension of the extracranial process [3]. They are typically seen arising from dura and less frequently may show intrapaenchymal or spine involvement.

The etiology of infantile fibromatosis is unknown so far. However, effects of maternal estrogen and familial association have been hypothesized [4].

Radiological studies are not characteristic due to variable appearances but it is very important in assessing the extent of the disease [5]. It usually appears as enhancing extra axial lesion with calcifications. It does not show restriction on diffusion weighted imaging in contrast due to highly cellular tumors like atypical teratoid rhabdoid tumor and primitive neuroectodermal tumors. Since the condition is not commonly known it may mimic malignant tumors. Definite diagnosis depends on histology.

The current is unique due to isolated posterior fossa involvement with minimal transjugular extension. Al-

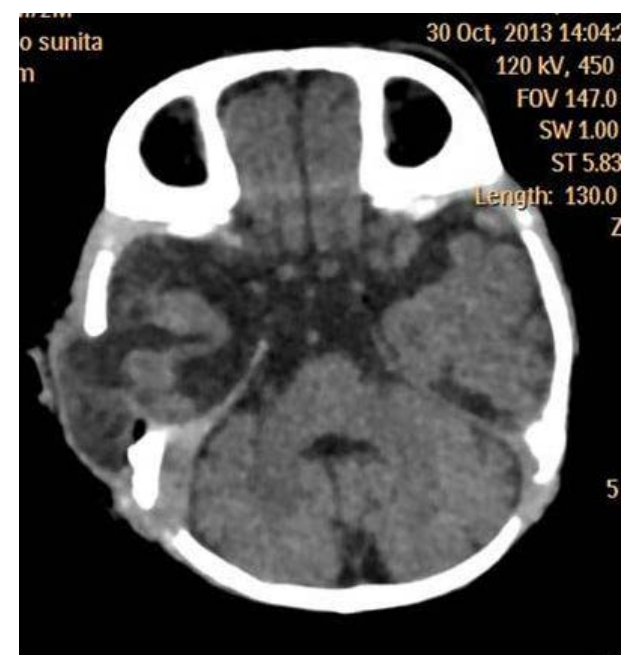

Figure 6. Follow-up plain CT head reveals significant reduction in size of lesion with post operative changes. 
though it is rare clinical entity, it should be considered in differential diagnosis of intracranial space occupying lesions.

Treatment algorithm is still indefinite. Surgical excision is advocated by some as primary treatment regimen for all types of disease. However, spontaneous regression has been reported. Others advocate routine follow up with surgical excision of lesions which adversely affect vital function. In our case, the lesion was causing focal neurological deficit due to its critical location in form of facial palsy. Hence, subtotal excision was performed. Recurrence rate is high in excised lesions reaching upto $10 \%-31 \%$ according to literature. Chemotherapeutic agents have been shown by some groups to be effective in life threatening complications and unresectable diseases [4].

To conclude we report a case of isolated posterior fossa infantile myofibromatosis. Though a rare entity, it should be considered in the differential of posterior fossa lesions in infancy especially when confined to the extraaxial compartment.

\section{Acknowledgements}

We would like to acknowledge the work of Suresh Dhakate, MD, Anand Hatgaonkar, MD in preparation of this case report.

\section{Conflicts of Interest}

There is no conflict in author or acknowledged persons.

\section{References}

[1] Chapman, P.R., Judd, C.D., Felgenhauer, J.L., Gruber, D.P. and Mornin, D. (2005) Infantile Myofibromatosis of the Posterior Fossa. AJR, 184, 1310-1312. http://dx.doi.org/10.2214/ajr.184.4.01841310

[2] Stout, A.P. (1954) Juvenile Fibromatosis. Cancer, 7, 953-978. http://dx.doi.org/10.1002/1097-0142(195409)7:5<953::AID-CNCR2820070520>3.0.CO;2-W

[3] Adickes, E.D., Goodrich, P., Auch Moedy, J., et al. (1985) Central Nervous System Involvement in Congenital Visceral Fibromatosis. Pediatric Pathology, 3, 329-340. http://dx.doi.org/10.3109/15513818509078793

[4] Gandhi, M.M., Nathan, P.C., Weitzman, S. and Levitt, G.A. (2003) Successful Treatment of Life Threatening Generalized Infantile Myofibromatosis Using Low Dose Chemotherapy. Journal of Pediatric Hematology/Oncology, 25, 750-754. http://dx.doi.org/10.1097/00043426-200309000-00016

[5] Wong, M.S.C. and Kwan, E.Y.W. (2004) Congenital Infantile Myofibromatosis: A Case Report and Review of Literature. HK J Paeditr (New Series), 9, 162-166. 